\title{
QUALIDADE PÓS-COLHEITA DE GOIABAS 'BRANCA DE KUMAGAI', TRATADAS COM CLORETO DE CÁLCIO'
}

\author{
RENATO VASCONCELOS BOTELHO ${ }^{2}$, NILTON LUIZ DE SOUZA ${ }^{3}$, \\ NATÁLIA APARECIDA RODRIGUES PERES ${ }^{4}$
}

\begin{abstract}
RESUMO - Com o objetivo de estudar o efeito do tratamento pós-colheita com cloreto de cálcio, pelo método da temperatura diferenciada, na conservação e qualidade de goiabas 'Branca de Kumagai', conduziu-se experimento em que frutos, na temperatura de $26^{\circ} \mathrm{C}$, foram imersos em soluções de cloreto de cálcio a $5^{\circ} \mathrm{C}$, nas concentrações de $0 \% ; 0,5 \% ; 1,5 \% ; 2,5 \%$ e $3,5 \%$. A testemunha foi representada por frutos que não receberam qualquer tipo de aplicação. Após a imersão, os frutos foram mantidos à temperatura ambiente. O tratamento na concentração de $0,5 \%$ de cloreto de cálcio estendeu em 34,8\% (3,2 dias) o período de conservação dos frutos, reduziu a taxa respiratória e a perda de massa fresca, aumentou ligeiramente os teores de sólidos solúveis totais, sem alterar a acidez titulável e o teor de ácido ascórbico. O tratamento a $0 \%$ de cloreto de cálcio reduziu a conservação e aumentou a taxa respiratória dos frutos, indicando que a temperatura da solução de $5^{\circ} \mathrm{C}$, foi prejudicial ao metabolismo dos frutos.
\end{abstract}

Termos para indexação - Psidium guajava, nutrição mineral, conservação, amadurecimento.

\section{POST-HARVEST QUALITY OF GUAVAS 'BRANCA DE KUMAGAI', TREATED WITH CALCIUM CHLORIDE}

\begin{abstract}
A trial was conducted to verify the effect of post-harvest treatment with calcium chloride by the temperature differential method on the shelf-life and quality of guavas 'Branca de Kumagai'. The treatments consisted in dipping fruits with pulp temperature at $26^{\circ} \mathrm{C}$ in solutions at $5^{\circ} \mathrm{C}$ of different calcium chloride concentrations: $0 \%, 0,5 \%, 1,5 \%, 2,5 \%$ e $3,5 \%$. The control did not receive any kind of application. After dipping, fruits were kept under environmental conditions. The treatment with $0,5 \%$ of calcium chloride extended the shelf-life in 34,8\% (3,2 days), decreased respiration rate and fresh weight loss, slightly increased the total soluble solids content, but not change the acid ascorbic content and the tritable acidity. The treatment at $0 \%$ of calcium chloride decreased its shelflife, indicating that the solution temperature of $5^{\circ} \mathrm{C}$ was harmful to the fruit metabolism.
\end{abstract}

Index terms - Psidium guajava, mineral nutrition, shelf-life, ripening.

\section{INTRODUÇÃO}

A goiaba 'Branca de Kumagai' é a cultivar de mesa mais cultivada no Estado de São Paulo. Apresentando frutos brancos e firmes, é bastante adequada para o consumo 'in natura', tanto para o mercado interno como para a exportação (Piza Jr. \& Kavati, 1994). Entretanto, segundo Akamine \& Goo (1979), por serem frutos climatéricos, as goiabas passam pelo processo de amadurecimento rapidamente e entram logo em senescência, o que reduz o seu período de conservação.

O papel do cálcio em retardar a maturação e a senescência em frutos e hortaliças tem sido relatado por vários autores. $\mathrm{O}$ cálcio tem um papel especial na manutenção da estrutura da parede e membrana celular, ativação de enzimas e interação com fitoormônios (Bangerth, 1979; Gleen \& Poovaiah, 1990; D'auzac, 1994; Picchioni et al., 1998).

Em goiabas 'Sardar', Singh et al. (1981) verificaram que a imersão dos frutos em solução a $1 \%$ de nitrato de cálcio proporcionou menor perda de massa fresca, redução da taxa respiratória e manutenção das qualidades organolépticas dos frutos por mais de 6 dias, enquanto aqueles não tratados se mantiveram em condições aceitáveis para o consumo por apenas 3 dias.

Em mangas 'Julie' tratadas com solução de cloreto de cálcio pela metodologia da temperatura diferenciada (frutos a $28^{\circ} \mathrm{C}$ e solução a $5^{\circ} \mathrm{C}$ ), Mootoo (1991) verificou aumento no período de conservação de 5 para 14 dias, menor perda de massa fresca e de firmeza, e desenvolvimento mais lento da coloração da casca. Segundo Betts \& Bramlage (1977), o maior incremento do teor de cálcio dos frutos através do tratamento de maçãs com solução de cloreto de cálcio a baixa temperatura foi devido a uma contração dos gases dos espaços intercelulares.

Em goiabas 'Branca de Kumagai', Carvalho et al. (1998) constataram aumento do teor de cálcio na polpa e epiderme dos frutos através da aplicação de cloreto de cálcio em solução à temperatura de $48^{\circ} \mathrm{C}$. Entretanto, os frutos apresentaram aparência externa prejudicada pela presença de manchas marrons na epiderme, e a polpa com aspecto gelatinoso e de coloração creme.

1 (Trabalho 086/2001). Recebido: 13/04/2001. Aceito para publicação: 08/02/2002. Parte da dissertação de mestrado do primeiro autor apresentada ao curso de pós-graduação em agronomia da FCA-UNESP, campus de Botucatu-SP. Trabalho financiado pela FAPESP.

2 Eng $^{\circ} \mathrm{Agr}^{\circ}$ M. Sc. Centro de Fruticultura, Instituto Agronômico de Campinas. Caixa Postal 28,CEP 13001-970, Campinas-SP, rebotelho@hotmail.com

$3 \mathrm{Eng}^{\mathrm{O}} \mathrm{Agr}^{\circ}$ Dr. Departamento de Proteção Vegetal, FCA-UNESP, Campus de Botucatu, C.P. 237,CEP 18603-970, Botucatu-SP.

4 Enga Agra M. Sc.Departamento de Proteção Vegetal, FCA-UNESP, Campus de Botucatu-SP. 
Baseando-se nos benefícios do cálcio na conservação de frutos, este trabalho objetivou estudar os efeitos do tratamento pós-colheita com cloreto de cálcio pela metodologia da temperatura diferenciada, em goiabas 'Branca de Kumagai' .

\section{MATERIAL E MÉTODOS}

As goiabas da cultivar Branca de Kumagai foram obtidas em pomar comercial, com 14 anos de idade, localizado no município de Campinas-SP. Os frutos foram colhidos no estádio de maturação verde-escuro, segundo Ahwalat et al. (1980). Posteriormente, estes foram selecionados, proporcionando amostras homogêneas, sendo em seguida lavados em água e secos ao ar.

O delineamento experimental foi inteiramente casualizado, com 10 repetições para as avaliações não destrutivas e 3 repetições para as avaliações destrutivas. As parcelas experimentais das avaliações não destrutivas e destrutivas foram constituídas de um e três frutos, respectivamente.

Para a aplicação pós-colheita de cloreto de cálcio, foi utilizada a metodologia da Temperatura Diferenciada descrita por Mootoo (1991). Após a lavagem e secagem dos frutos, a temperatura da polpa foi estabilizada em $26^{\circ} \mathrm{C}$ através da manutenção dos frutos em incubadora B.O.D. à mesma temperatura. Depois de 24 horas da colheita, os frutos foram imersos em solução de cloreto de cálcio diidratado $\left(\mathrm{CaCl}_{2} .2 \mathrm{H}_{2} \mathrm{O}\right.$, com $76 \%$ de $\mathrm{CaCl}_{2}$ ) e $0,1 \%$ de espalhante adesivo Tween $20 \mathrm{a}$ a $5^{\circ} \mathrm{C}$, por 2 horas. Em seguida, os frutos foram retirados da solução, secos ao ar, e colocados em caixas de papelão telescópica e mantidos sob condições ambiente (temperatura média de $25,3^{\circ} \mathrm{C}$ e umidade relativa média de $71,2 \%$ ).

Os tratamentos consistiram de 5 concentrações de $\mathrm{CaCl}_{2} \cdot 2 \mathrm{H}_{2} \mathrm{O}(0 \%, 0,5 \%, 1,5 \%, 2,5 \%$ e $3,5 \%)$ e a testemunha foi representada por frutos que não receberam qualquer tipo de aplicação.

\section{Amostra destrutiva}

Do homogeneizado de três frutos de cada uma das repetições, foram realizadas as seguintes análises:

1.Teor de sólidos solúveis totais: utilizando-se de refratômetro de mesa ATAGO-3T, com autocompensação de temperatura (Lutz, 1985).

2. Acidez total titulável: uma alíquota de $5 \mathrm{~g}$ da polpa em $100 \mathrm{ml}$ de água destilada foi titulada com $\mathrm{NaOH} 0,1 \mathrm{~N}$, utilizandose de três gotas de fenolftaleína $10 \mathrm{~g} . \mathrm{L}^{-1}$ como indicador, sendo os resultados expressos em porcentagem de ácido cítrico (Lutz, 1985).

3. Teor de ácido ascórbico: o conteúdo de ácido ascórbico (após a oxidação a ácido deidroascórbico) foi determinado pelo método colorímetro com 2,4 dinitrofenil hidrazina, segundo Pearson (1976). A leitura foi realizada em espectrofotômetro e os resultados expressos em $\mathrm{mg} / 100 \mathrm{~g}$ de polpa.

Estas análises foram realizadas aos $0 ; 3 ; 6$ e 9 dias após a aplicação de cloreto de cálcio.

\section{Amostra não destrutiva}

Os frutos da amostra não destrutiva foram pesados diariamente para o cálculo da perda de massa fresca acumulada expressa em porcentagem, assim como estabeleceu-se o número de dias em cada fruto que permaneceu em condições aceitáveis para comercialização.

Os frutos foram considerados impróprios para comercialização quando apresentavam sintomas de podridões, como depressões e frutificações de patógenos na epiderme, ou amolecimento acentuado da polpa, verificado pela deformação sofrida pelos frutos durante o seu manuseio.

Avaliou-se também a alteração da coloração da epiderme através da observação visual, conferindo a cada fruto notas, numa escala de 1 a 5 , de acordo com os índices sugeridos por Ahlawat et al. (1980): 1-verde-escuro; 2-verde-escuro início verdeclaro; 4-verde-claro início verde-amarelo-claro; e 5-verde-amareloclaro. Para a análise estatística, comportaram-se os dias necessários para cada fruto atingir a nota máxima 5 .

Para a determinação da taxa respiratória, realizada diariamente, três frutos foram retirados aleatoriamente de cada tratamento e colocados individualmente, por uma hora, em campânula de vidro, sob fluxo contínuo de ar isento de $\mathrm{CO}_{2}$. A taxa respiratória expressa $\mathrm{em} \mathrm{mg} \mathrm{de} \mathrm{CO}_{2} \cdot \mathrm{kg}^{-1} \cdot \mathrm{h}^{-1}$ foi determinada através da titulação de solução de $\mathrm{KOH}$ a $0,1 \mathrm{~N}$, com solução de $\mathrm{HCl} 0,1 \mathrm{~N}$, calculando-se o peso de $\mathrm{CO}_{2}$ liberado pela respiração dos frutos, seguindo metodologia adaptada de Bleinroth et al. (1976).

Os resultados foram submetidos a análises de variância para experimento inteiramente casualizado, e teste de Tukey, ao em nível de $5 \%$ de probabilidade, excetuando-se os dados de taxa respiratória, para os quais se estudou-se a regressão polinomial.

\section{RESULTADOS E DISCUSSÃO}

Para os dados de período de conservação apresentados na Tabela 1, embora não tenha havido diferenças significativas entre os tratamentos e a testemunha, a aplicação de cloreto de cálcio, a 0,5\%, aumentou em 34,8\% (3,2 dias) o período de conservação. Resultados semelhantes foram constatados por Mootoo (1991) em mangas, e por Singh et al. (1981) em goiabas. Para a coloração da epiderme, esta tendência voltou a se repetir, ou seja, os frutos do tratamento a $0,5 \%$ de cloreto de cálcio atingiram a coloração máxima (índice 5), em média, 1,6 dia após a testemunha.

Quando se utilizou apenas água, sem adição de cloreto de cálcio, o período de conservação foi, em média, de apenas 6,6 dias, sendo significativamente inferior ao tratamento com cálcio na concentração de $0,5 \%$. Possivelmente, houve um efeito prejudicial da baixa temperatura $\left(5^{\circ} \mathrm{C}\right)$, causando um tipo de "chilling injury". Observando-se a Figura 1, nota-se que a taxa respiratória destes frutos permaneceu alta. Alterações na taxa respiratória e, portanto, no metabolismo dos fruto, provocadas por baixas temperaturas, foram relatadas por Castro \& Sigrist (1991) em goiabas híbridas (brancas).

Nos tratamentos em que se adicionou o cálcio, percebese que o efeito prejudicial do frio foi anulado. Chaplin \& Scott (1980) também verificaram redução de incidência de "chilling injury" em abacates tratados com cloreto de cálcio.

Constatou-se ainda que o incremento na concentração 
de cálcio não proporcionou aumento na conservação dos frutos. Singh et al. (1981) também verificaram melhores resultados em termos de conservação pós-colheita de goiabas com soluções a $0,5 \%$ e $1,0 \%$ de cloreto de cálcio, enquanto as concentrações de $1,5 \%$ e $2,0 \%$ foram menos efetivas.

Conway et al. (1995) sugerem que as paredes celulares se saturam de íon cálcio, ou seja, há um limite de sítios de ligação. Desta forma, maiores concentrações de cloreto de cálcio na solução resultam em aumento no conteúdo total de cálcio livre, porém há um menor aumento de cálcio ligado à parede celular, podendo resultar em injúrias aos frutos, além de fitotoxidez.

Os fatores que contribuíram para reduzir o período de conservação dos frutos foram o amolecimento da polpa e a ocorrência de podridões. Os patógenos causadores de podridões foram identificados como sendo Macrophoma sp e Colletotrichum gloeosporioides. A manutenção da firmeza da polpa e a menor ocorrência de podridões nos frutos tratados com cálcio podem estar relacionadas ao papel deste cátion em ligar os componentes pécticos da parede celular, principalmente na lamela média (Gleen \& Poovaiah, 1990). Além disso, o cálcio aumentaria a estabilidade das membranas, principalmente por atrasar o processo catabólico dos componentes lipídicos, mantendo o turgor das células (Picchioni et al., 1998).

Analisando ainda os dados apresentados na Figura 1, observa-se que todos os tratamentos com cálcio reduziram a taxa respiratória, principalmente na fase pré-climatérica, ou seja, até os 4 dias. $\mathrm{O}$ tratamento na concentração de $0,5 \%$ possibilitou também um atraso no pico climatérico, que ocorreu somente no $12^{\circ}$ dia após imersão dos frutos, quando a taxa respiratória atingiu $55,52 \mathrm{mg} \cdot \mathrm{kg}^{-1} \cdot \mathrm{h}^{-1}$. Segundo Bangerth et al. (1979), a redução da respiração, observada em frutos tratados com cálcio, poderia ser parcialmente explicada pelas alterações na permeabilidade das membranas celulares, assim como pelo seu efeito direto nas enzimas respiratórias.

Embora não tenha havido diferenças significativas entre os tratamentos em termos de porcentagem de perda de massa fresca acumulada, observa-se, na Tabela 2 , uma tendência de menor perda de massa fresca dos frutos tratados a $0,5 \%$ e $1,5 \%$ de cloreto de cálcio, estando de acordo com os relatos de outros autores (Singh et al.,1981; Mootoo, 1991). Por outro lado, os tratamentos a $3,5 \%$ e $2,5 \%$ provocaram maior perda de massa fresca, refletindo o efeito prejudicial de doses mais elevadas de cloreto de cálcio.

Para os dados das análises de acidez total titulável e teor de ácido ascórbico (Tabelas 4 e 5), não houve diferenças significativas entre os tratamentos pós-colheita e a testemunha. Nota-se, entretanto, que o teor de sólidos solúveis totais da testemunha e do tratamento na concentração de $0 \%$ de cloreto de cálcio apresentou valores ligeiramente inferiores no $9^{\circ}$ dia de avaliação (Tabela 3). Segundo Singh et al. (1981), isto pode ocorrer em função do processo mais acelerado de oxidação dos açúcares devido às maiores taxas respiratórias.

TABELA 1 - Período de conservação (dias) e tempo para atingir coloração máxima (índice 5) de goiabas 'Branca de Kumagai' imersas em soluções a diferentes concentrações de cloreto de cálcio, pelo método da temperatura diferenciada.

\begin{tabular}{|c|c|c|}
\hline Tratamentos & Conservação (dias) & Coloração máxima (dias) \\
\hline Tes te munha & $9,2 a b^{1}$ & 7,0 \\
\hline $0 \% \mathrm{CaCl}_{2}$ & $6,6 \mathrm{~b}$ & 6,6 \\
\hline $0,5 \% \mathrm{CaCl}_{2}$ & $12,4 \mathrm{a}$ & 8,6 \\
\hline $1,5 \% \mathrm{CaCl}_{2}$ & $10,0 \mathrm{ab}$ & 8,2 \\
\hline $2,5 \% \mathrm{CaCl}_{2}$ & $9,6 \mathrm{ab}$ & 7,0 \\
\hline $3,5 \% \mathrm{CaCl}_{2}$ & $9,8 \mathrm{ab}$ & 7,0 \\
\hline CV (\%) & 27,98 & 31,60 \\
\hline DMS $5 \%$ & 5,25 & 4,57 \\
\hline
\end{tabular}

${ }^{1}$ Médias seguidas da mesma letra, numa mesma coluna, não diferem entre si, pelo teste de Tukey, ao nível de $5 \%$ de probabilidade.

TABELA 2 - Porcentagem de perda de massa fresca acumulada de goiabas 'Branca de Kumagai' imersas em soluções a diferentes concentrações de cloreto de cálcio, pelo método da temperatura diferenciada.

\begin{tabular}{|c|c|c|c|c|c|c|c|c|c|c|}
\hline \multirow[t]{2}{*}{ Tratamentos } & \multicolumn{9}{|c|}{ Dias após imersão } & \multirow[b]{2}{*}{ Médias } \\
\hline & 1 & 2 & 3 & 4 & 5 & 6 & 7 & 8 & 9 & \\
\hline Testemunha & 1,00 & 2,17 & 2,58 & 3,37 & 4,16 & 4,94 & 6,17 & 7,15 & 9,11 & $4,51 \mathrm{ab}$ \\
\hline $0 \% \mathrm{CaCl}_{2}$ & 0,87 & 1,07 & 2,16 & 3,15 & 4,05 & 4,67 & 5,61 & 6,73 & 8,40 & $4,08 \mathrm{~b}$ \\
\hline $0,5 \% \mathrm{CaCl}_{2}$ & 0,73 & 1,16 & 2,03 & 2,91 & 3,39 & 4,35 & 5,09 & 6,29 & 7,27 & $3,70 \mathrm{~b}$ \\
\hline $1,5 \% \mathrm{CaCl}_{2}$ & 0,61 & 1,02 & 1,97 & 2,81 & 3,58 & 4,35 & 5,22 & 6,11 & 7,63 & $3,70 \mathrm{~b}$ \\
\hline $2,5 \% \mathrm{CaCl}_{2}$ & 0,80 & 1,72 & 2,64 & 3,44 & 4,37 & 5,28 & 6,18 & 7,96 & 9,26 & $4,65 \mathrm{ab}$ \\
\hline $3,5 \% \mathrm{CaCl}_{2}$ & 1,13 & 1,96 & 3,11 & 4,23 & 5,36 & 6,60 & 8,04 & 8,73 & 9,50 & $5,41 \mathrm{a}$ \\
\hline Mé dias & $0,86 \mathrm{~h}$ & $1,52 \mathrm{gh}$ & $2,41 \mathrm{fg}$ & $3,32 \mathrm{ef}$ & $4,15 \mathrm{de}$ & $5,03 \mathrm{~cd}$ & $6,05 b c$ & $7,16 a b$ & $8,52 \mathrm{a}$ & \\
\hline C.V. (\%) & 29,44 & & & & & & & & & \\
\hline Fatores & Trat: & mentos & & & trat & nentos $\mathrm{x}$ & mpo & & & \\
\hline Teste F & 7,1 & & 74 , & $5 * *$ & & $0,254^{\text {n.s. }}$ & & & & \\
\hline
\end{tabular}

${ }^{1}$ Médias seguidas da mesma letra, numa mesma coluna, ou linha não diferem entre si, pelo teste de Tukey, ao nível de 5\% de probabilidade. 
TABELA 3 - Teor de sólidos solúveis totais (\%) de goiabas ‘Branca de Kumagai’ imersas em soluções a diferentes concentrações de cloreto de cálcio, pelo método da temperatura diferenciada.

\begin{tabular}{|c|c|c|c|c|c|}
\hline Tratamentos & \multicolumn{5}{|c|}{ D ias após imersão } \\
\hline Testemunha & 7,86 a $\mathrm{A} \mathrm{B}^{1}$ & 8,10 a $\mathrm{A}$ & 7,30 b $\quad$ A B & 7,07 b B & - \\
\hline $0 \% \quad C$ a C $1_{2}$ & 7,86 a A B & 8,40 a $\mathrm{A}$ & $7,93 \mathrm{abcAB}$ & $7,15 \mathrm{ab} B$ & - \\
\hline $0,5 \% \quad \mathrm{Ca} \mathrm{C}_{2}$ & 7,86 a A & 8,13 a A & $8,27 \mathrm{ab} \quad \mathrm{A}$ & $7,97 \mathrm{ab} \mathrm{A}$ & 7,53 \\
\hline $2,5 \% \quad \mathrm{Ca} \mathrm{Cl}_{2}$ & 7,86 a A & $7,90 \mathrm{a} \mathrm{A}$ & $7,80 \mathrm{abcA}$ & $7,50 \mathrm{ab} \mathrm{A}$ & - \\
\hline $\begin{array}{l}3,5 \% \text { C a C } 1_{2} \\
\mathrm{C} \mathrm{V}(\%)\end{array}$ & $\begin{array}{c}7,86 \text { a B } \\
5,21\end{array}$ & 7,77 a B & $8,77 \mathrm{a} \quad \mathrm{A}$ & 7,90 a b B & - \\
\hline Fatores & tratamentos & te m p o & \multicolumn{2}{|c|}{$\begin{array}{c}\text { Tratamentos } \mathrm{x} \text { tempo } \\
2,968^{* *}\end{array}$} & \\
\hline
\end{tabular}

${ }^{1}$ médias seguidas da mesma letra, minúscula na mesma coluna, ou da mesma letra maiúscula na mesma linha, não diferem entre si, pelo teste de Tukey, ao nível de $5 \%$ de probabilidade.

TABELA 4 - Acidez total titulável (\%) de goiabas ‘Branca de Kumagai’ imersas em soluções a diferentes concentrações de cloreto de cálcio, pelo método da temperatura diferenciada.

\begin{tabular}{|c|c|c|c|c|c|}
\hline \multirow{2}{*}{ Tratamentos } & \multicolumn{5}{|c|}{ Dias a pós imersão } \\
\hline & $\mathbf{0}$ & 3 & 6 & 9 & 12 \\
\hline Testemunha & 0,481 & 0,608 & 0,560 & 0,579 & - \\
\hline $0 \% \quad C$ a $C \mathbf{I}_{2}$ & 0,481 & 0,667 & 0,586 & 0,586 & - \\
\hline $0,5 \%$ C a C $\mathrm{I}_{2}$ & 0,481 & 0,566 & 0,611 & 0,530 & 0,571 \\
\hline $1,5 \%$ C a C $1_{2}$ & 0,481 & 0,536 & 0,530 & 0,577 & - \\
\hline $2,5 \% \quad C$ a C l 2 & 0,481 & 0,560 & 0,592 & 0,584 & - \\
\hline $3,5 \% \quad \mathrm{C}$ a C $\mathrm{I}_{2}$ & 0,481 & 0,563 & 0,610 & 0,608 & - \\
\hline $\begin{array}{l}\text { M é di a s } \\
C V(\%)\end{array}$ & $\begin{array}{c}0,481 \mathrm{~b}^{1} \\
8,45\end{array}$ & $0,573 \mathrm{a}$ & $0,582 \mathrm{a}$ & $0,574 \mathrm{a}$ & \\
\hline Fatores & tratamentos & te m po & \multirow{2}{*}{\multicolumn{2}{|c|}{$\begin{array}{c}\text { Tratam e ntos } \mathrm{x} \text { te } \mathrm{m} \text { po } \\
0,845 \text { n.s. }\end{array}$}} & \\
\hline Teste $F$ & $0,821^{\mathrm{n} . \mathrm{s} .}$ & $18,859 * *$ & & & \\
\hline
\end{tabular}

${ }^{1}$ Médias seguidas da mesma letra, na mesma linha, não diferem entre si, pelo teste de Tukey, ao nível de 5\% de probabilidade.

TABELA 5 - Teor de ácido ascórbico (mg/100g) de goiabas ‘Branca de Kumagai’ imersas em soluções a diferentes concentrações de cloreto de cálcio, pelo método da temperatura diferenciada.

\begin{tabular}{|c|c|c|c|c|c|}
\hline \multirow[t]{2}{*}{ Tratamentos } & \multicolumn{5}{|c|}{ D ias após imersão } \\
\hline & $\mathbf{0}$ & 3 & 6 & 9 & 12 \\
\hline Testemunha & 66,2 & 96,4 & 123,6 & 139,9 & - \\
\hline $0 \% \mathrm{CaC} \mathrm{l}_{2}$ & 66,2 & 110,1 & 131,6 & 135,3 & - \\
\hline $0,5 \% \mathrm{C}$ a C $\mathrm{I}_{2}$ & 66,2 & 80,8 & 115,9 & 156,8 & 152,6 \\
\hline $1,5 \% \mathrm{Ca} \mathrm{Cl}_{2}$ & 66,2 & 92,2 & 106,8 & 124,3 & - \\
\hline $2,5 \% \mathrm{Ca} \mathrm{Cl}_{2}$ & 66,2 & 99,0 & 109,3 & 105,1 & - \\
\hline $3,5 \% \mathrm{Ca} \mathrm{Cl}_{2}$ & 66,2 & 92,6 & 135,7 & 118,7 & - \\
\hline M édias & $66,2 \mathrm{c}$ & $95,20 \mathrm{~b}$ & $120,09 \mathrm{a}$ & $130,02 \mathrm{a}$ & \\
\hline CV $(\%)$ & 20,21 & & & & \\
\hline Fatores & tratamentos & tempo & \multicolumn{2}{|c|}{ tratamentos $\times$ tempo } & \\
\hline Teste F & $0,947^{\text {n.s. }}$ & $33,833 * *$ & \multicolumn{2}{|c|}{$0,966^{\text {n.s. }}$} & \\
\hline
\end{tabular}

${ }^{1}$ Médias seguidas da mesma letra, na mesma linha, não diferem entre si, pelo teste de Tukey, ao nível de $5 \%$ de probabilidade.

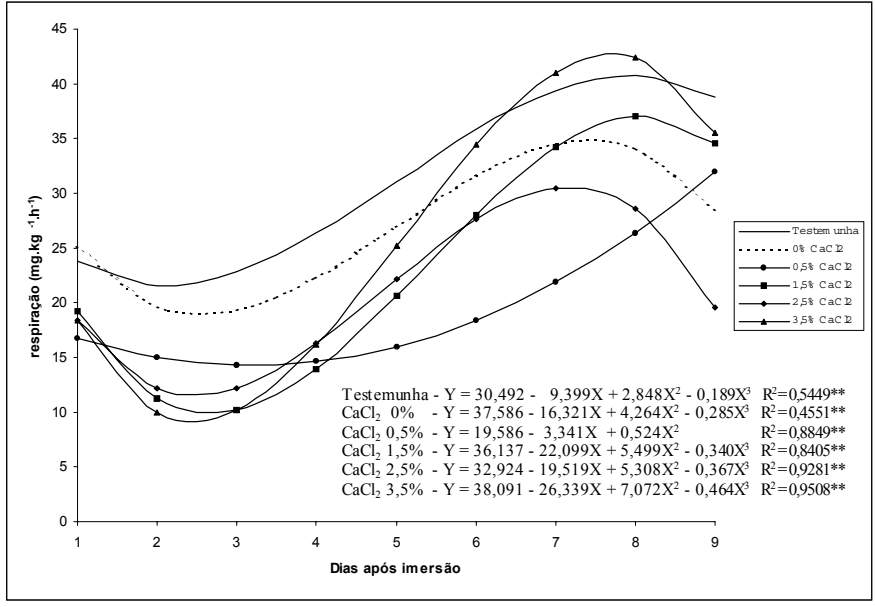

FIGURA 1 - Taxa respiratória $\left(\mathrm{mg} \mathrm{CO}_{2} \cdot \mathrm{kg}^{-1} \cdot \mathrm{h}^{-1}\right)$ de goiabas 'Branca de Kumagai' imersas em soluções a diferentes concentrações de cloreto de cálcio, pelo método da temperatura diferenciada.

\section{CONCLUSÕES}

1. Os frutos de goiaba 'Branca de Kumagai' são sensíveis à baixa temperatura quando tratados em solução a $5^{\circ} \mathrm{C}$, por 2 horas, levando a um distúrbio fisiológico, ou seja, um tipo de "chilling injury", verificado pelo aumento nas taxas respiratórias e menor conservação pós-colheita.

2. Entre os efeitos dos tratamentos pós-colheita com cálcio em goiabas 'Branca de Kumagai', destacam-se: aumento do período de conservação pós-colheita, redução da taxa respiratória e redução da suscetibilidade dos frutos a distúrbios fisiológicos causados por baixas temperaturas.

3. Entre as doses de cloreto de cálcio testadas, a solução a $0,5 \%$ foi a mais eficiente, sendo que doses mais elevadas reduziram a conservação pós-colheita dos frutos. 


\section{AGRADECIMENTOS}

Agradecimento à FAPESP, pelos recursos disponibilizados para o desenvolvimento desta pesquisa.

\section{REFERÊNCIAS BIBLIOGRÁFICAS}

AHLAWAT, V.P.; YAMDAGNI, R.; JINDAL, D.C. Studies on the effect of post-harvest treatments on storage behavior of guava (Psidium guajava L.) cv. Sardar (L-49). Haryana Agricultural University Journal of Research, Hissar v.10, n.2, p.244-247, 1980.

AKAMINE, E.K.; GOO, T. Respiration and ethylene production in fruits of species and cultivars of Psidium and species of Eugenia. Journal of the American Society for Horticultural Science, Alexandria, v.104, n.5 p.632-635, set.1979.

BANGERTH, F. Calcium related physiological disorders of plants. Annual Reviews of Phytopathology, Palo Alto, v.17, p.91-122, 1979.

BETTS, H.A.; BRAMLAGE, W.J. Uptake of calcium apples from postharvest dips in calcium chloride solutions. Journal of the American Society for Horticultural Science, Alexandria, v.102, n.6, p.785-788, nov.1977.

BLEINROTH, E.W.; ZUCHINI, A.G.; POMPEU, R.M. Determinação das características físicas e mecânicas de variedades de abacates e sua conservação pelo frio. Coletânea do Ital, Campinas, v.7, n. 1, p.29-81, 1976.

CARVALHO, H. A.; CHITARRA, M.I.F.; CHITARRA, A.B., MENEZES, J.B. Eficiência da concentração de cloreto de cálcio e do tempo de imersão no tratamento pós-colheita de goiaba de polpa branca cv. Kimagai. Revista Brasileira de Fruticultura, Jaboticabal, v.20, n.3, p.375-381, dez. 1998.

CASTRO, J.V.; SIGRIST, J.M.M. Matéria-prima. In: MEDINA, J.C. et al. Goiaba: Cultura, matéria-prima, processamento e aspectos econômicos. 2. ed. Campinas: ITAL, 1991. cap.2, p.121139. (Frutas Tropicais, 6)

CHAPLIN, G.R.; SCOTT, K.J. Association of calcium in chilling injury susceptibility of stored avocados, HortScience, Alexandria, v.14, n.4, p.514-515, ago.1980.

CONWAY, W.S.; SAMS, C.E.; WATADA, A.E. Relationship between total and cell wall bound calcium in apples following postharvest pressure infiltration of calcium chloride. Acta Horticulturae, Tokyo, n.398, p.31-39, mar.1995.

D'AUZAC, J. Le calcium: un messager dans la responsé des plants aux stimuli. Plantations, Recherche, Développement: Fruit, Paris, v.1, n.1, p.27-27, 1994.

GLEEN, G.M.; POOVAIAH, B.W. Calcium-mediated postharvest changes in texture and cell wall structure and composition in Golden Delicious apples. Journal of the American Society for Horticultural Science, Alexandria, v.115, n.6, p.962-968, nov. 1990.

LUTZ, A. Normas analíticas do Instituto Adolfo Lutz: métodos químicos para análise de alimentos. 3. ed. São Paulo, SP; Coordenadoria dos serviços técnicos especializados - Secretaria do Estado de Saúde, 1985. 533p.

MOOTOO, A. Effect of post-harvest calcium chloride dips on ripening changes in 'Julie' mangoes. Tropical Science,Surrey, v.31, n.3, p.243-248, 1991.

PERSON, D.; COX, H.E. The chemical analisis of foods. New York: Chem. Publ., 1976.393p.

PICCHIONI, G.A.; WATADA, A.E.; CONWAY, W.S.; WHITAKER, B.D.; SAMS, C.E. Postharvest calcium infiltration delays membrane lipid catabolism in apple fruit. Journal of Agricultural and Food Chemistry, Columbus, v.46, n.7, p.24522457, jul.1998.

PIZA JR., C.T., KAVATI, R. A cultura da goiaba de mesa. Campinas: CATI, 1994.28p. (Boletim Técnico, 219)

SINGH, B.P.; SINGH, H.K., CHAUAN, K.S. Effect of post-harvest calcium treatments on the storage life of guava fruits. Indian Journal of Agricultural Science, New Delhi, v.51, n.1, p.44-47, jan.1981. 